\title{
Crystal Structure of (Z)-1-[4-(Trifluoromethyl)benzylidene]thiosemicarbazide
}

\author{
Uwaisulqarni M. Osman, ${ }^{* \dagger}$ Azieda Syafika N. Farizal, ${ }^{*}$ Suhana ArShad, ${ }^{* *}$ and \\ Maisara Abdul KADIR* \\ *School of Fundamental Science, Universiti Malaysia Terengganu, 21030 Kuala Terengganu, Terengganu, \\ Malaysia \\ **X-ray Crystallography Unit, School of Physics, Universiti Sains Malaysia, 11800, USM Pulau Pinang, \\ Malaysia
}

\begin{abstract}
The compound of (Z)-1-[4-(trifluoromethyl)benzylidene]thiosemicarbazide crystallizes in the triclinic space group $P \overline{1}$ and $Z=2$ with cell parameters $a=7.0109(8) \AA, b=11.7862(13) \AA, c=14.9095(16) \AA, \alpha=111.814(2)^{\circ}, \beta=91.720(2)^{\circ}$, $\gamma=101.036(2)^{\circ}$ and $V=1115.7(2) \AA^{3}$. The crystal structure was solved by direct methods and refined by full-matrix least-squares on $F^{2}$ to final values of $R 1=0.0438$ and $w R 2=0.1078$. In the crystal, each molecule is linked by intermolecular $\mathrm{N}-\mathrm{H}$...S hydrogen bonds.
\end{abstract}

(Received October 11, 2016; Accepted November 18, 2016; Published on web January 10, 2017)

Thiosemicarbazide derivatives are known to be potentially used as antibacterial agents, ${ }^{1,2}$ analytical reagents for the analysis of metals, ${ }^{3}$ optical storage ${ }^{4}$ and absorbents. ${ }^{5}$ These applications may be explained by the fact that the thiosemicarbazone usually acts as chelating ligands, by which bonding can take place through the sulfur atom and hydrazine nitrogen. Previously, the crystal structure of the isomer (Z)-1-[2-(trifluoromethyl)benzylidene]thiosemicarbazide had already been published. ${ }^{6}$ However, the present crystal structure of $(Z)-1-[4-($ trifluoromethyl)benzylidene]thiosemicarbazide (1) was yet reported. Thus, our present report is to contribute deeper understanding about the crystal structure of (1). In addition, the effect of the trifluoromethyl group at the para position towards phenyl group is also discussed. Compound $\mathbf{1}$ (Fig. 1) was synthesized by the reaction between thiosemicarbazide with 2-(trifluoromethyl)benzaldehyde in ethanol, as reported in previous literature. ${ }^{?}$

Being colorless and having a plate-shape single crystal of $\mathbf{1}$ is suitable for X-ray diffraction analysis, which was obtained from slow evaporation in methanol at room temperature. Data collection and the refinement parameters are listed in Table 1. The hydrogen atoms that bounded to the nitrogen atoms were found from difference Fourier maps and refined freely [refined $\mathrm{N}-\mathrm{H}$ distance, $0.78(2)-0.85(2) \AA]$. The remaining $\mathrm{H}$ atoms were positioned geometrically $[\mathrm{C}-\mathrm{H}=0.93 \AA]$ and refined using a riding model with $U_{\text {iso }}(\mathrm{H})=1.2 U_{\text {eq }}(\mathrm{C})$. All fluorine atoms are disordered over two orientations, with a final refined occupancy

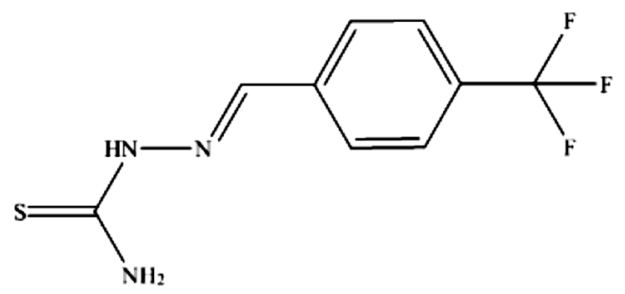

Fig. 1 Chemical diagram of the title compound (1).

$\dagger$ To whom correspondence should be addressed. E-mail: uwais@umt.edu.my ratio of $0.676(15): 0.324(15)$. The disordered atoms were subjected to a similarity restraint (SAME), except for atoms F1X, F2X and F3X. Additionally, the similar-ADP restraint (SIMU) and rigid-bond restraint (DELU) was applied to the F1B, F2B, F3B, F1X, F2X and F3X atoms in the molecule.

In with respect to the title compound, $\mathbf{1}$ is structurally similar to (Z)-1-[2-(trifluoromethyl)benzylidene]thiosemicarbazide, except for the presence of a trifluoromethyl group at the para position attached to a phenyl group. ${ }^{6}$ An ORTEP drawing of the two molecules in the asymmetric unit is shown in Fig. 2. Molecules 1 show a cis conformation between the N1A-N2A and $\mathrm{N} 3 \mathrm{~A}-\mathrm{C} 9 \mathrm{~A}$ bonds through the N2A-C9A bond. However,

Table 1 Crystal data and experimental data

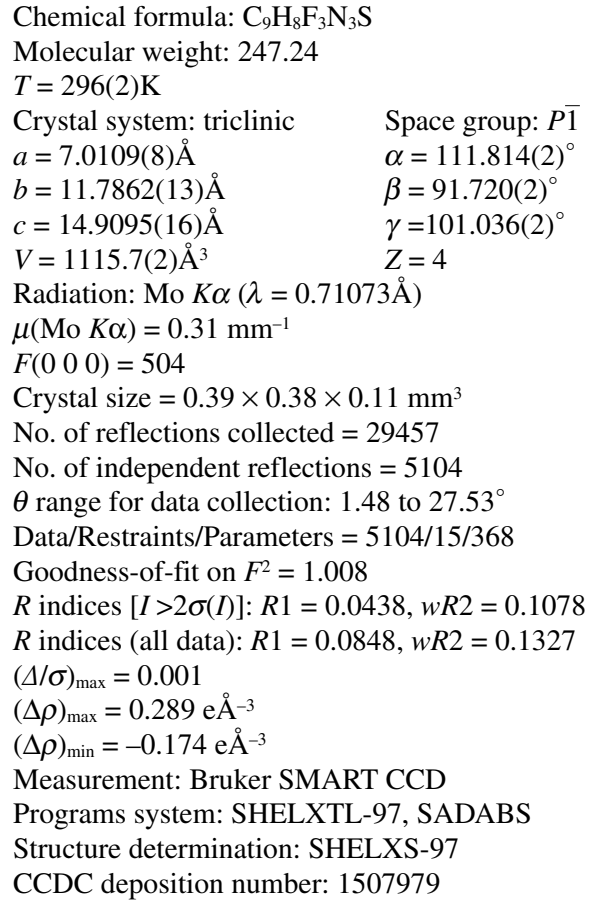


Table 2 Selected geometric parameters, bond length and angles $\left(\AA,{ }^{\circ}\right)$

\begin{tabular}{ccll}
\hline Bond & Bond length/A & \multicolumn{1}{c}{ Bond } & \multicolumn{1}{c}{ Bond angle/ $^{\circ}$} \\
\hline C8A-N1A & $1.272(3)$ & C5A-C8A-N1A & $122.30(2)$ \\
N1A-N2A & $1.372(2)$ & C8A-N1A-N2A & $115.21(17)$ \\
N2A-C9A & $1.339(2)$ & N1A-N2A-C9A & $121.06(17)$ \\
C9A-N3A & $1.311(2)$ & N2A-C9A-N3A & $118.09(18)$ \\
C9A-S1A & $1.690(18)$ & N2A-C9A-S1A & $123.34(16)$ \\
C8B-N1B & $1.265(2)$ & C5B-C8B-N1B & $121.60(2)$ \\
N1B-N2B & $1.373(2)$ & C8B-N1B-N2B & $116.49(17)$ \\
N2B-C9B & $1.340(2)$ & N1B-N2B-C9B & $120.01(17)$ \\
C9B-N3B & $1.308(2)$ & N2B-C9B-N3B & $117.28(17)$ \\
C9A-S1A & $1.685(18)$ & N2B-C9B-S1B & $119.47(14)$ \\
\hline
\end{tabular}

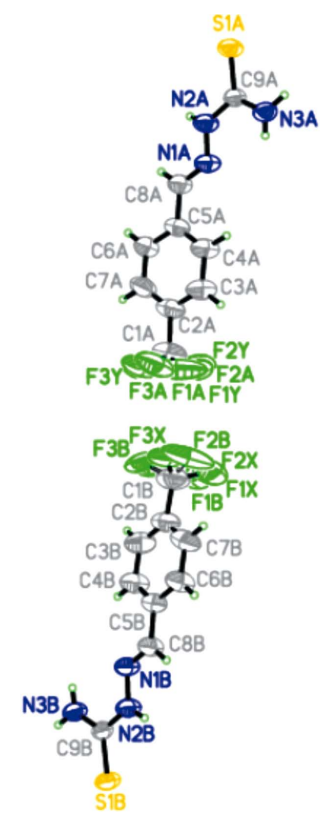

Fig. 2 ORTEP structure of two molecules in the asymmetric unit for (Z)-1-[4-(trifluoromethyl)benzylidene]thiosemicarbazide, showing $50 \%$ probability ellipsoids.

both the N1A-N2A and C9A-S1A bonds are in a trans conformation through the N2A-C9A bond. In addition, another asymmetric unit maintains the conformations. The plane (defined by $\mathrm{S} 1 \mathrm{~A} / \mathrm{N} 1 \mathrm{~A} / \mathrm{N} 2 \mathrm{~A} / \mathrm{N} 3 \mathrm{~A} / \mathrm{C} 2 \mathrm{~A} / . . . / \mathrm{C} 9 \mathrm{~A})$ for the first residue is almost planar with a maximum deviation of $-0.034(3) \AA$ for atom $\mathrm{C} 2 \mathrm{~A}$, as compared with the second residue plane (defined by $\mathrm{N} 1 \mathrm{~B} / \mathrm{C} 1 \mathrm{~B} / . . . / \mathrm{C} 8 \mathrm{~B}$ ) with a maximum deviation of $0.046(5) \AA$ for atom $\mathrm{C} 1 \mathrm{~B}$, and makes a dihedral angle of $21.51(9)^{\circ}$. Both thioureido (defined by S1B/N2B/N3B/C9B) and $\mathrm{N} 1 \mathrm{~B} / \mathrm{C} 1 \mathrm{~B} / . . . / \mathrm{C} 8 \mathrm{~B}$ fragments for the second residue have a maximum deviation of $0.046(5) \AA$ for atom $\mathrm{C} 1 \mathrm{~B}$, and make dihedral angles of $19.34(11)^{\circ}$. The double-bond lengths for C8A-N1A and C8B-N1B are 1.272(3) and 1.265(2) , respectively. This is slightly shorter compared with $\mathrm{C}=\mathrm{N}$ (1.279(3) $\AA$ ) in the other isomer. ${ }^{6}$ Meanwhile, the bond angles for C8A-N1A-N2A and C8B-N1B-N2B are 115.21(17) and $116.49(17)^{\circ}$, respectively, and have no significant differences with another reported isomer, which has a bond angle of $116.0(2)^{\circ} .6$ Other bond lengths and angles are in the normal ranges. ${ }^{8}$ This study reveals that the position of the trifluoromethyl group at either ortho or para of phenyl does not have any significant effects towards the bond length and angles of the

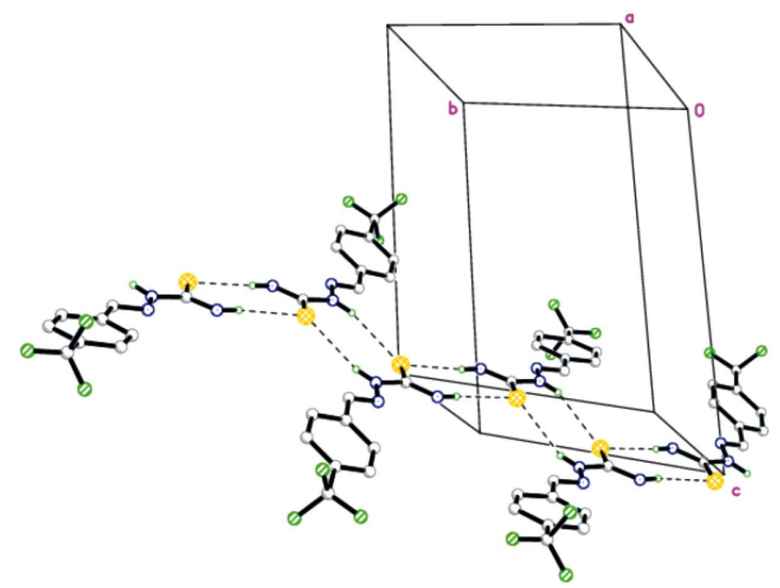

Fig. 3 Packing of structure 1, showing molecules connected by $\mathrm{N}-\mathrm{H}-\mathrm{S}$ S hydrogen bonds (dashed lines).

Table 3 Hydrogen-bond geometry $\left(\AA{ }^{\circ}\right)$

\begin{tabular}{|c|c|c|c|c|}
\hline D-H...A & D-H & $\mathrm{H} \cdot \cdots \mathrm{A}$ & 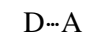 & $\mathrm{D}-\mathrm{H} \cdots \mathrm{A}$ \\
\hline N2A-H1NA $\cdots$ S1A & 0.81 & 2.60 & 3.3513 & 155 \\
\hline N3A-H3NA-..S1B ${ }^{\mathrm{ii}}$ & 0.87 & 2.59 & 3.4505 & 173 \\
\hline N3B-H3NB-..S1A & 0.85 & 2.49 & 3.3295 & 171 \\
\hline N2B-H1NB-..S1B & 0.85 & 2.59 & 3.4168 & 165 \\
\hline
\end{tabular}

Symmetry codes: (i) $3-x, 1-y, 2-z$; (ii) $3+x, y, 1+z$; (iii) $-3+x, y$, $-1+z$; (iv) $-1-x, 2-y,-z$.

$\mathrm{C}=\mathrm{N}-\mathrm{N}$ moiety. The introduction of trifluoromethyl at the para position of benzyl forbids the formation of intramolecular hydrogen bonds, as similarly observed in the case of $(Z)-1-[2-$ (trifluoromethyl)benzylidene]thiosemicarbazide. ${ }^{6}$ In the crystal structure of $\mathbf{1}$, each of molecule is linked by four intermolecular

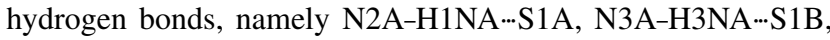
N3B-H3NB-..S1A and N2B-H1NB-..S1B (symmetry codes as in Table 3) to form a one-dimensional wave-like chain parallel to (032) plane (Fig. 2).

\section{Acknowledgements}

The authors thank the Ministry of Higher Education of Malaysia (MoHE) for a research grant (FRGS 59387), Universiti Sains Malaysia and Universiti Malaysia Terengganu; School of Fundamental Science for financial and research supports.

\section{References}

1. R. J. Nevagi, A. S. Dhake, H. I. Narkhede, and P. Kaur, Bioorg. Chem., 2014, 54, 68.

2. T. A. Yousef, O. K. Alduaij, A. F. Ahmed, G. M. A. E. Reash, and O. A. E. Gammal, J. Mol. Struct., 2016, 1119, 351.

3. K. Anita and N. R. Singh, Spectrochim. Acta, Part A, 2011, 81, 117.

4. J. T. J. Prakash and J. M. S. Gnanaraj, Spectrochim. Acta, Part A, 2015, 135, 25.

5. Y. Xiong, L. Wan, J. Xuan, Y. Wang, Z. Xing, W. Shan, and Z. Lou, J. Hazard Mater, 2016, 310, 277.

6. X. Chen and Z.-L. Jing, Acta Crystallogr., 2011, E67, o3369.

7. R. Kothari and B. Sharma, Int. J. Appl. Biol. Pharm. Tech., 2013, 3, 176.

8. F. K. Allen, O. Kennard, and D. G. Watson, J. Chem. Soc, Perkin Trans. 2, 1987, 12, S1. 